\title{
可见光催化烯烃砜基化启动的远程醛基碳-氢键直接硫化反应
}

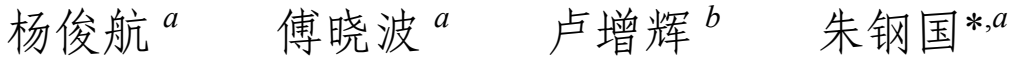 \\ ( ${ }^{a}$ 浙江师范大学化学系先进催化材料教育部重点实验室 金华 321004) \\ $\left({ }^{b}\right.$ 重庆市万州区生态环境监测站 万州 404000)
}

\begin{abstract}
摘要 有机硫化合物广泛应用于医药、农药、新材料等领域，因此，发展新的碳-硫键形成方法非常重要. 近年来，烯 烃的自由基砜基化反应作为一种温和、有效的有机硫化合物合成策略得到了快速发展，其中，烯烃的硫砜基化反应因 为能够同时构建两种不同的碳-硫键成了一种非常有吸引力的碳-硫键形成方法. 以硫代磺酸酯同时作砜基化和硫化试 剂，实现了一个可见光催化烯烃砜基化启动的远程醛基碳-氢键直接硫化反应，一步合成了 6-或 7-砜基取代的硫酯类化 合物. 反应具有优秀的原子经济性，产率中等到良好，能兼容各种不同的官能团. 相比传统的烯烃 1,2-或 1,1-硫砜基化 反应，首次实现了官能团化烯烃的远程硫砜基化反应，拓展了现有硫砜基化反应方法学. 初步的机理研究表明，该反 应可能经历一个可见光催化的自由基反应历程.
\end{abstract}

关键词 可见光催化; 砜基化; 硫化; 硫代磺酸酯; 远程碳-氢键官能团化

\section{Visible-Light Photocatalytic Remote Thiolation of Aldehydes Triggered by Sulfonylation of Alkenes With Thiosulfonates}

\author{
Yang, Junhang $^{a} \quad \mathrm{Fu}$ Xiaobo $^{a} \quad$ Lu, Zenghui $^{b} \quad$ Zhu, Gangguo ${ }^{*, a}$ \\ ( ${ }^{a}$ Key Laboratory of the Ministry of Education for Advanced Catalysis Materials, Department of Chemistry, \\ Zhejiang Normal University, Jinhua 321004) \\ ( ${ }^{b}$ Wanzhou Ecological Environment Monitoring Station, Chongqing 404000)
}

\begin{abstract}
Due to the prevalence of organosulfur compounds in pharmaceuticals, agrochemicals, and functional materials, the development of new efficient and practical methods for the construction of $\mathrm{C}-\mathrm{S}$ bonds is highly desirable in organic synthesis. Recently, the radical sulfonylation of alkenes has attracted considerable attention because of its efficient and versatile synthesis of organosulfur compounds under mild reaction conditions. The previous methods usually involve the formation of one $\mathrm{C}-\mathrm{S}$ bond. In contrast, the thiosulfonylation of alkenes represents a highly attractive protocol for the concurrent formation of two distinct $\mathrm{C}-\mathrm{S}$ bonds. Herein, a novel visible-light photocatalytic remote thiolation of aldehydes triggered by the radical sulfonylation of unactivated alkenes has been developed, with readily available thiosulfonates as both the sulfonating and thiolating reagents, successfully giving 6- or 7-sulfonylated thioesters in moderate to high yields with broad substrate scope and excellent atom-economics. As compared to the traditional methods that are limited to 1,2- or 1,1-thiosulfonylation of alkenes, the reaction described here constitutes the first example of 1,6- or 1,7-thiosulfonylation of functionalized alkenes, thus offering a good complementary protocol to the existing methods. Preliminary mechanistic studies suggest a radical pathway consisting of the formation of sulfonyl radical, alkene sulfonylation, intramolecular 1,n-hydrogen atom transfer $(1, n$-HAT), and thiolation of acyl radical. A representative procedure for the visible-light induced remote thiolation of aldehydes initiated by the sulfonylation of alkenes with thiosulfonates is as following: To a mixture of thiosulfonates 2 $(0.5 \mathrm{mmol})$, $\operatorname{Ir}(\mathrm{ppy})_{3}(1 \mathrm{~mol} \%)$, and $\mathrm{K}_{2} \mathrm{HPO}_{4}(0.5 \mathrm{mmol})$ in $4 \mathrm{~mL}$ of MeCN was added alkenyl aldehydes $1(0.25 \mathrm{mmol})$ under a $\mathrm{N}_{2}$ atmosphere. After $18 \mathrm{~h}$ of irradiation with $15 \mathrm{~W}$ blue LEDs at $25{ }^{\circ} \mathrm{C}$, the reaction mixture was quenched with water, extracted with EtOAc, dried over anhydrous $\mathrm{Na}_{2} \mathrm{SO}_{4}$, concentrated, and purified by column chromatography with silica gel $($ EtOAc/petroleum ethers $=1: 5)$ to give products 3 or 4 .
\end{abstract}

Keywords visible-light photocatalytic; sulfonylation; thiolation; thiosulfonate; remote $\mathrm{C}-\mathrm{H}$ bond functionalization

\section{1 引言}

硫原子在医药、农药和功能材料分子中广泛存在. 有机硫化合物常常具有显著的生物活性 ${ }^{[1 \sim 4]}$, 例如, 依
立曲坦(Eletriptan)是一种高选择性的 5- $\mathrm{HT}_{1 \mathrm{~B} / 1 \mathrm{D}}$ 受体激动 剂, 可用于治疗偏头痛(图 1) ${ }^{[1]} ; \operatorname{Sch} 42427$ 是一种抗真菌 药物 ${ }^{[2]}$; 氟替卡松(Fluticasone) 是一种糖皮质激素类药 物，具有良好的抗炎和抗过敏作用，能有效减轻哮喘症

* E-mail: gangguo@zjnu.cn; Tel.: 0579-82282640; Fax: 0579-82282269

Received May 2, 2019; published June 12, 2019.

Supporting information for this article is available free of charge via the Internet at http://sioc-journal.cn.

Project supported by the National Natural Science Foundation of China (No. 21672191).

项目受国家自然科学基金(No. 21672191)资助. 
状 $^{[3]}$. 另一方面, 砜基化合物具有丰富的化学反应性质, 能顺利发生 Julia 烯基化 ${ }^{[5]}$ 、亲电取代 ${ }^{[6]}$ 、碳-硫键的还 原断裂 ${ }^{[7]}$ 及光催化和氟代 ${ }^{[8]}$ 等反应. 可见, 砜基等含硫 有机化合物的合成非常重要 ${ }^{[9]}$.<smiles>CN1CCC[C@H]1Cc1c[nH]c2ccc(CCS(=O)(=O)c3ccccc3)cc12</smiles>

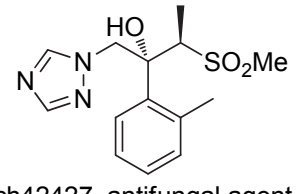
Sch42427, antifungal agent

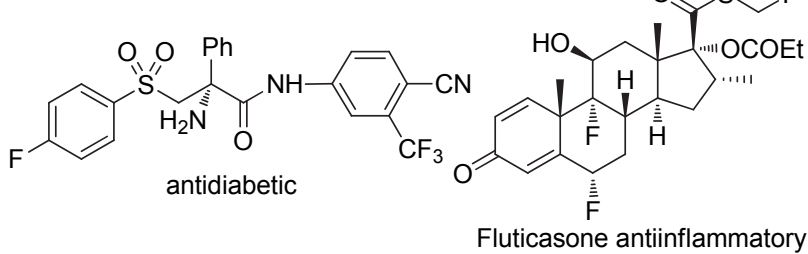

图 1 部分含有砜基或硫酯的生物活性分子

Figure 1 Selected bioactive sulfone- or thioester-containing molecules

随着自由基化学的发展，烯烃的自由基砜基化反应 成了合成砜基化合物的有效方法. 烯烃的氢砜基化 ${ }^{[10]}$ 、 脱氢或脱羧砜基化 ${ }^{[11]}$ 、杂原子砜基化 ${ }^{[12]}$ 、碳砜基化 ${ }^{[13]}$ 或砜基化启动的自由基环化反应 ${ }^{[14]}$ 相继被报道. 最近, 烯烃的硫砜基化反应因为能够同时构建两种不同的 $\mathrm{C}-$ $S$ 键引起了化学工作者的关注 ${ }^{[15 ~ 17]}$. 例如, 吕龙和沈其 龙等 ${ }^{[15]}$ 首次实现了非活泼烯烃的 1,2-硫二氟甲基砜基化 和 1,2-硫单氟甲基砜基化反应(图 2a). 几乎同时, 徐政虎 等 ${ }^{[16 a]}$ 发展了一个金催化和可见光催化协同催化的芳基 烯烃的 1,2-硫砜基化反应(图 2b). 吴劼课题组 ${ }^{[17]}$ 采用二 氧化硫插入策略, 实现了三组分的烯烃 1,2-硫砜基化反 应(图 2c). 之后, 徐政虎课题组 ${ }^{[16 b]}$ 又实现了铜催化芳基 烯烃的 1,1-硫砜基化反应(图 2d). 需要指出的是, 尽管 烯烃的 1,2-或 1,1-硫砜基化反应已经取得重要进展, 烯 烃的远程硫砜基化反应仍然没有实现.

我们课题组 ${ }^{[18]}$ 近年来开展了远程醛基的直接官能 才化反应研究, 例如, 最近发展了一个烯烃氟烷基化引 发的远程醛基的直接芳基化反应 ${ }^{[18 b]}$. 在这些工作基础 上, 我们采用硫代磺酸酯同时作砜基化和硫化试剂, 实 现了一个可见光催化 ${ }^{[19]}$ 的烯烃砜基化引发的远程醛基 碳-氢键直接硫化反应，一步合成了 6-或 7-砜基取代的 硫代羧酸酯 (图 2e), 为含有多种不同碳-硫键的有机化 合物提供了温和、高效的合成方法.

\section{2 结果与讨论}

\section{1 反应条件优化}

首先, 采用 2-烯丙基苯甲醛(1a)和苯基硫代苯磺酸 酯(2a)为模板底物对反应条件进行了篮选. 当以 $\operatorname{Ir}(p p y)_{3}$ 为光催化剂, $\mathrm{K}_{2} \mathrm{CO}_{3}$ 做碱, 乙腈为反应溶剂, $15 \mathrm{~W}$ 蓝色 LEDs 灯照射 $18 \mathrm{~h}$, 以 $60 \%$ 的产率得到 1,6-硫砜基 (a) Shen and Lu's work: 1,2-thiosulfonylation of alkenes<smiles></smiles>

(b) Xu's work: 1,2-thiosulfonylation of alkenes

$$
\mathrm{Ar} \curvearrowright+\mathrm{ArSO}_{2} \mathrm{SR} \underset{\mathrm{DCE}, \text { blue LEDs }}{\stackrel{\mathrm{IPrAuCl}, \mathrm{AgSbSF}_{6}}{\mathrm{Ru}(\mathrm{bpy})_{3} \mathrm{Cl}_{2}}}
$$

(c) Wu's work: 1,2-thiosulfonylation of alkenes

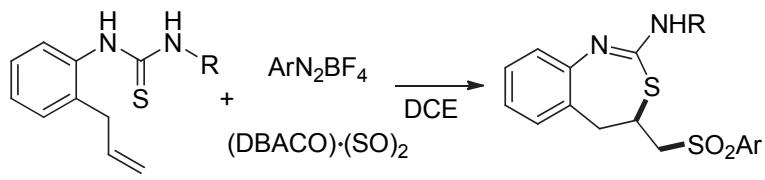

(d) Xu's work: 1,1-thiosulfonylation of alkenes

$$
\mathrm{Ar} \curvearrowright \mathrm{ArSO}_{2} \mathrm{SR} \frac{\mathrm{CuTC}, \text { bpy }}{\text { air, } 50^{\circ} \mathrm{C}} \rightarrow
$$

(e) This work: 1,6- or 1,7-thiosulfonylation of functionalized alkenes

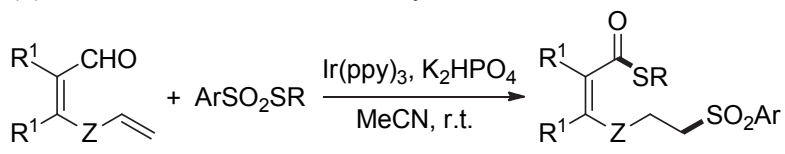

图 2 烯烃的硫砜基化反应

Figure 2 Thiosulfonylation of alkenes

表 1 反应条件优化 ${ }^{a}$

Table 1 Optimization of reaction conditions

\begin{tabular}{|c|c|c|c|c|}
\hline Entry & $\mathrm{PC}$ & Base & Solvent & Yield $/ \%$ \\
\hline 1 & $\operatorname{Ir}(\mathrm{ppy})_{3}$ & $\mathrm{~K}_{2} \mathrm{CO}_{3}$ & $\mathrm{MeCN}$ & 60 \\
\hline 2 & $\operatorname{Ir}(\text { ppy })_{3}$ & $\mathrm{Na}_{2} \mathrm{CO}_{3}$ & $\mathrm{MeCN}$ & 40 \\
\hline 3 & $\operatorname{Ir}(p p y)_{3}$ & $\mathrm{Et}_{3} \mathrm{~N}$ & $\mathrm{MeCN}$ & 45 \\
\hline 4 & $\operatorname{Ir}(p p y)_{3}$ & KOAc & $\mathrm{MeCN}$ & 46 \\
\hline 5 & $\operatorname{Ir}(p p y)_{3}$ & $\mathrm{~K}_{3} \mathrm{PO}_{4}$ & $\mathrm{MeCN}$ & 44 \\
\hline 6 & $\operatorname{Ir}(p p y)_{3}$ & $\mathrm{~K}_{2} \mathrm{HPO}_{4}$ & $\mathrm{MeCN}$ & 71 \\
\hline 7 & $\mathrm{Ru}(\mathrm{bpy})_{3} \mathrm{Cl}_{2}$ & $\mathrm{~K}_{2} \mathrm{HPO}_{4}$ & $\mathrm{MeCN}$ & 30 \\
\hline 8 & $\operatorname{Ir}\left[\mathrm{dF}\left(\mathrm{CF}_{3}\right) \mathrm{ppy}\right]_{2}(\mathrm{dtbpy}) \mathrm{PF}_{6}$ & $\mathrm{~K}_{2} \mathrm{HPO}_{4}$ & $\mathrm{MeCN}$ & 54 \\
\hline 9 & $\operatorname{Ir}(\text { ppy })_{3}$ & $\mathrm{~K}_{2} \mathrm{HPO}_{4}$ & DMF & 23 \\
\hline 10 & $\operatorname{Ir}(p p y)_{3}$ & $\mathrm{~K}_{2} \mathrm{HPO}_{4}$ & DMSO & 40 \\
\hline 11 & $\operatorname{Ir}(p p y)_{3}$ & $\mathrm{~K}_{2} \mathrm{HPO}_{4}$ & THF & 40 \\
\hline 12 & $\operatorname{Ir}(p p y)_{3}$ & $\mathrm{~K}_{2} \mathrm{HPO}_{4}$ & DCM & 68 \\
\hline 13 & $\operatorname{Ir}(p p y)_{3}$ & $\mathrm{~K}_{2} \mathrm{HPO}_{4}$ & $\mathrm{MeCN} / \mathrm{H}_{2} \mathrm{O}^{b}$ & 61 \\
\hline 14 & $\operatorname{Ir}(p p y)_{3}$ & $\mathrm{~K}_{2} \mathrm{HPO}_{4}$ & $\mathrm{MeCN}^{c}$ & 79 \\
\hline 15 & $\operatorname{Ir}(\text { ppy })_{3}{ }^{d}$ & $\mathrm{~K}_{2} \mathrm{HPO}_{4}$ & $\mathrm{MeCN}$ & 57 \\
\hline 16 & - & $\mathrm{K}_{2} \mathrm{HPO}_{4}$ & $\mathrm{MeCN}$ & 0 \\
\hline 17 & $\operatorname{Ir}(\mathrm{ppy})_{3}$ & - & $\mathrm{MeCN}$ & 50 \\
\hline
\end{tabular}

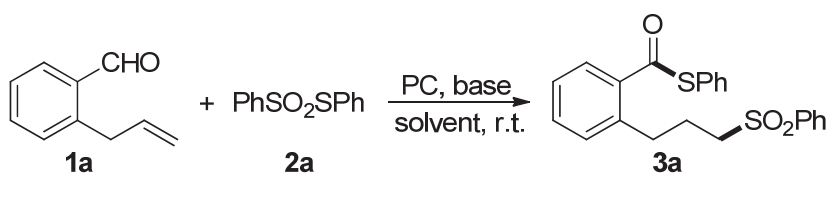

${ }^{a}$ Reaction conditions: 1a $(0.25 \mathrm{mmol}), 2 \mathrm{a}(0.5 \mathrm{mmol}), \mathrm{PC}(1 \mathrm{~mol} \%)$, base $(0.5$ mmol), solvent $(2 \mathrm{~mL})$, blue LEDs, $25{ }^{\circ} \mathrm{C}, 18 \mathrm{~h} .{ }^{b} \mathrm{~A} 1: 1$ mixture of MeCN and $\mathrm{H}_{2} \mathrm{O}$ was used. ${ }^{c} \mathrm{MeCN}(4 \mathrm{~mL})$ was used. ${ }^{d} \mathrm{Ir}(\mathrm{ppy})_{3}(0.5 \mathrm{~mol} \%)$ was used. 
化产物 3a(表 1, Entry 1). 受此鼓舞, 我们进一步研究了 反应的碱. 结果发现, $\mathrm{K}_{2} \mathrm{HPO}_{4}$ 有助于反应产率的提高, 达到了 71\% (Entries $2 \sim 6$ ). 其它光催化剂例如 $\mathrm{Ru}(\mathrm{bpy})_{3} \mathrm{Cl}_{2}$ 和 $\operatorname{Ir}\left[\mathrm{dF}\left(\mathrm{CF}_{3}\right) \mathrm{ppy}\right]_{2}$ (dtbpy) $\mathrm{PF}_{6}$ 则表现出了相 对较低的催化效果(Entries 7,8).

与此同时, 我们考察了溶剂对反应的影响, 发现二 氯甲烷(DCM)也是一种较为理想的溶剂, 但是, 其它溶 剂例如二甲基甲酰胺(DMF)、二甲基亚砜(DMSO)和四 氢呋喃(THF) 则导致产率明显下降(Entries 9 13). 令人 高兴的是, 适当降低反应浓度 $(4 \mathrm{~mL} \mathrm{MeCN}$ 进行反应)有 助于反应效率的提升, 以 $79 \%$ 的产率得到 3a (Entry 14). 控制实验表明, 光催化剂 $\operatorname{Ir}(\mathrm{ppy})_{3}$ 对反应的顺利进行是 必须的, 而碱的加入对产率提高有明显的促进作用 (Entries 16,17). 因此, 该反应的最佳条件为: 1a $(0.25$ $\mathrm{mmol}), 2 \mathrm{a}(0.5 \mathrm{mmol}), \mathrm{Ir}(\mathrm{ppy})_{3}(1 \mathrm{~mol} \%), \mathrm{K}_{2} \mathrm{HPO}_{4}(0.5$ $\mathrm{mmol}), \mathrm{MeCN}(4 \mathrm{~mL}), 15 \mathrm{~W}$ 蓝色 LEDs 灯照射下 $25{ }^{\circ} \mathrm{C}$ 搅拌反应 $18 \mathrm{~h}$.

\section{2 底物拓展}

确定最优反应条件之后, 我们对该光催化烯基醛的 远程硫砜基化反应适用范围进行了研究. 如表 2 所示, 该反应具有良好的普适性. 4-和 5-氯-2-烯丙基苯甲醛 $\mathbf{1 b}$ 和 $1 \mathrm{c}$ 分别以 $78 \%$ 和 74\% 的产率得到预期产物 $3 \mathrm{~b}$ 和 $3 \mathrm{c}$, 而 6-氯-2-烯丙基苯甲醛 1d 则只以 28\%产率得到远程硫 砜基化产物 3d, 说明醛基周围的立体环境对反应产率 有着较大影响. 尽管贫电子和富电子的苯甲醛都能顺利 进行该反应, 相对而言, 贫电子底物的产率有所下降 (3e, 3f vs. 3g $\sim 3 \mathbf{j})$.

烯烃上取代基的引入不利于反应的进行，例如 $\mathbf{1 m}$ 仅以 $50 \%$ 的产率得到相应产物 $3 \mathrm{~m}$, 这可能是碳自由基 位阻的增加不利于分子内氢迁移造成的. 与此相对应的 是, 烯丙位取代基的引入不会对反应产生不利影响(3n, 3o). 2-烯丙氧基苯甲醛(1p)也能顺利发生该反应, 以 $40 \%$ 的产率得到相应的 1,7-硫砜基化产物 3p, 说明除了 1,5-氢迁移(1,5-HAT) 外, 1,6-氢迁移也是可以发生的 ${ }^{[20]}$. 在该反应条件下, 吡啶甲醛衍生物 $\mathbf{1 q}$ 则不能得到预期 产物 (3q). 此外, 我们考察了非芳香醛的反应情况. 例 如, 环己烯基甲醛 $1 r$ 以中等的产率得到预期产物 3r, 而 1-己烯-5-醛 $1 \mathrm{~s}$ 则不能进行该反应( $3 \mathrm{~s})$, 这可能是 1,5-氢 迁移产生的酰基自由基缺乏邻位不饱和键的共轭稳定 作用造成的.

另一方面, 我们对硫代磺酸酯的适用范围进行了研 究. 如表 3 所示, 4-、3-和 2-氯苯基硫代苯磺酸酯 $\mathbf{2 b} \sim 2 \mathbf{2 d}$ 分别以 70\%、67\%和 74\%的产率得到预期产物 $4 \mathbf{a} \sim 4 \mathbf{c}$, 说明硫代磺酸酯的位阻对反应的影响不大. 此外, 烷基 硫代苯磺酸酯也能顺利进行反应, 得到相应的远程硫砜 基化产物 $(\mathbf{4 f} \sim \mathbf{4 h})$.

\section{3 机理研究}

接着, 我们对该反应的机理进行了研究. 在标准反
表 2 烯基醛的适用范围 $a$

Table 2 Scope of alkenyl aldehydes<smiles>C=CCc1ccccc1C=O</smiles>

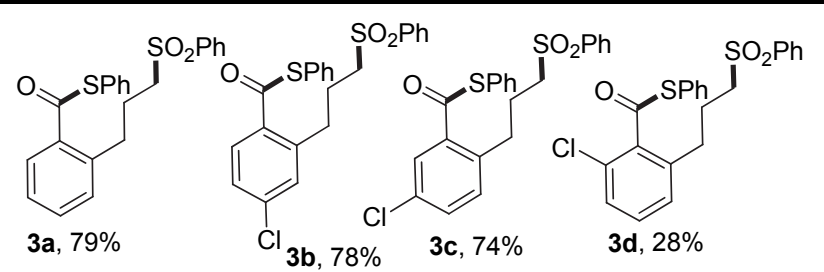

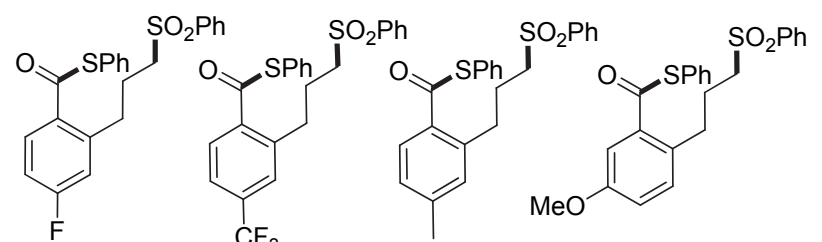

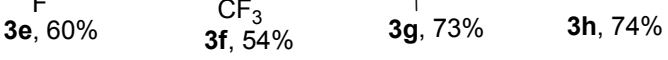

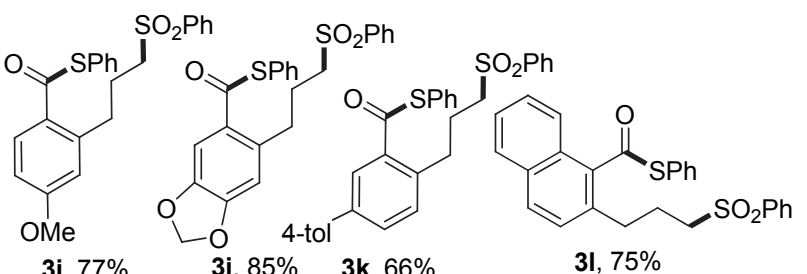

$3 \mathbf{i}, 77 \%$

3j, $85 \% \quad 3 \mathbf{k}, 66 \%$

31, $75 \%$<smiles>CC(CC[Se]c1ccccc1)c1ccccc1C(=O)[SnH2]c1ccccc1</smiles>

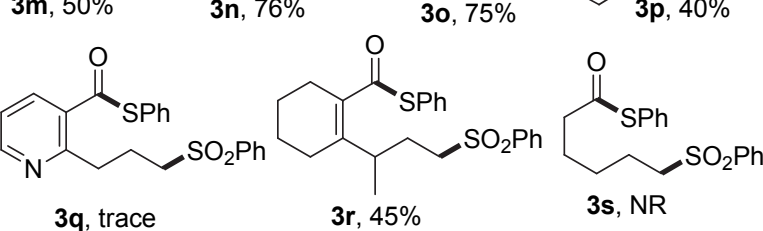

${ }^{a}$ Reaction conditions: $1(0.25 \mathrm{mmol}), 2 \mathrm{a}(0.5 \mathrm{mmol}), \operatorname{Ir}(\mathrm{ppy})_{3}(1 \mathrm{~mol} \%)$, $\mathrm{K}_{2} \mathrm{HPO}_{4}(0.5 \mathrm{mmol}), \mathrm{MeCN}(4 \mathrm{~mL})$, blue LEDs, $25{ }^{\circ} \mathrm{C}, 18 \mathrm{~h}$.

应条件下, 加入 2 equiv.的自由基捕获剂四甲基哌啶氧 化物(TEMPO)或 1,1-二苯乙烯，反应完全被抑制，以 42\%的产率分离到了 1,2-硫砜基化产物 5(图 3a, 3b), 这 些结果表明反应可能经历了自由基的反应历程. 反应体 系加入 2 equiv. 的甲醇, 以 $56 \%$ 的产率得到预期产物 3a, 而没有分离到相应的酯化产物 6(图 3c), 这说明酰基自 由基被氧化生成酰基正离子的可能性是比较小的. 开/ 关灯实验表明, 光照对标准反应的顺利进行是必须的 (图 3d), 说明自由基链反应的可能性是比较小的.

根据上述实验结果和前人的报道 ${ }^{[15,16]}$, 我们提出了 如下一个可能的反应机理(图 4). 首先, Ir(III)催化剂经 光照产生激发态的光催化剂 $\operatorname{Ir}(\mathrm{III})^{*}, \operatorname{Ir}(\mathrm{III})^{*}$ 再与 $\mathbf{2 a}$ 单电 子转移产生 $\mathrm{Ir}(\mathrm{IV}) 、 \mathrm{PhSO}_{2} \bullet$ 和 $\mathrm{PhS}^{-}$. 接着, 烯烃砜基化 随后发生一个分子内的 1,5-氢迁移产生酰基自由基 II. 
表 3 硫代磺酸酯的适用范围 ${ }^{a}$

Table 3 Scope of thiosulfonates

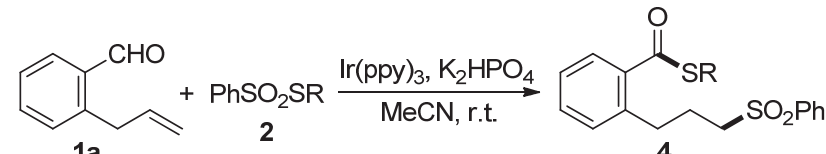<smiles>O=C(Sc1ccc(Cl)cc1)c1ccccc1CCC[Sb](=O)(O)Oc1ccccc1</smiles>

4a, $70 \%$<smiles>Cc1ccc(SC(=O)c2ccccc2CCCOS(=O)(=O)c2ccccc2)cc1</smiles>

4d, $66 \%$

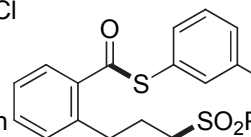

4b, $67 \%$

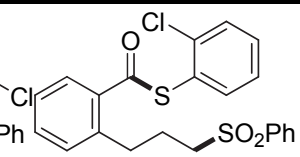<smiles>COc1ccc(SC(=O)c2ccccc2CCCS(=O)(=O)Oc2ccccc2)cc1</smiles>

4 e, $55 \%$

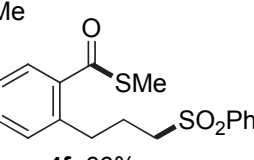

4f, $66 \%$<smiles>CCCc1ccccc1C(=O)OCC</smiles>

4g, $63 \%$<smiles>O=C(SCCCc1ccccc1C(=O)SOc1ccccc1)c1ccccc1</smiles>

$4 \mathrm{~h}, 56 \%$
${ }^{a}$ Reaction conditions: 1a $(0.25 \mathrm{mmol}), 2(0.5 \mathrm{mmol}), \operatorname{Ir}(\mathrm{ppy})_{3}(1 \mathrm{~mol} \%)$, $\mathrm{K}_{2} \mathrm{HPO}_{4}(0.5 \mathrm{mmol}), \mathrm{MeCN}(4 \mathrm{~mL})$, blue LEDs, $25{ }^{\circ} \mathrm{C}, 18 \mathrm{~h}$.

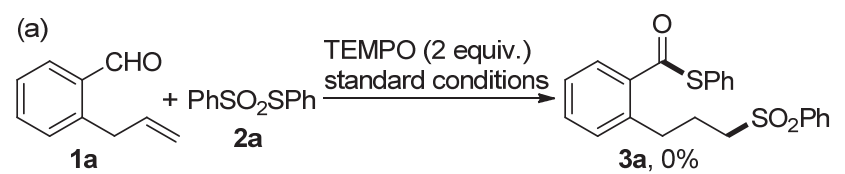

(b)<smiles>C=CCc1ccccc1C=O</smiles><smiles>C=CCc1ccccc1C=O</smiles>

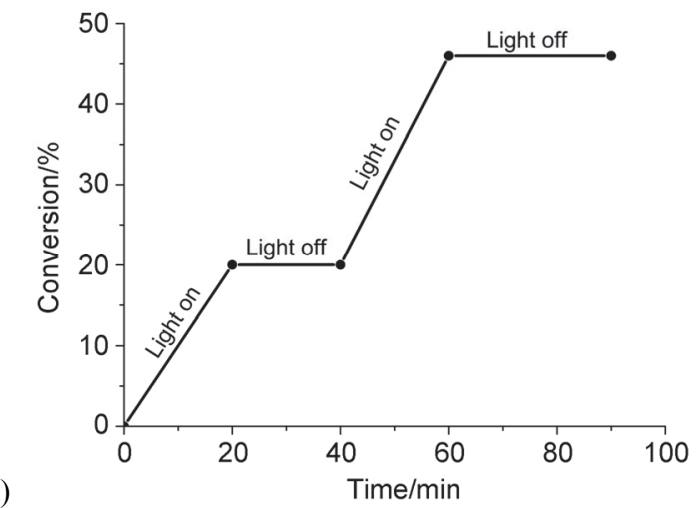

图 3 机理研究

Figure 3 Mechanistic studies

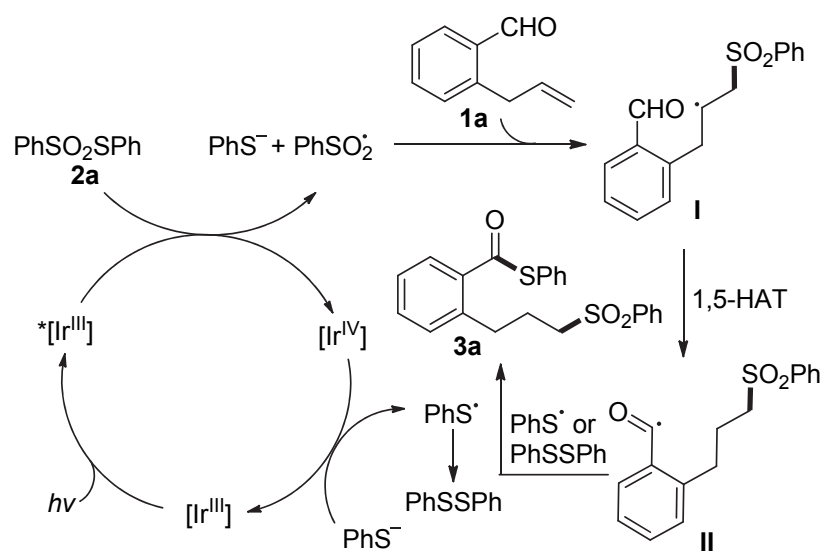

图 4 可能的反应机理

Figure 4 Plausible mechanism

与此同时, $\mathrm{PhS}^{-}$被现场生成的 $\operatorname{Ir}(\mathrm{IV})$ 氧化产生 $\mathrm{PhS} \cdot$, 然 后，它与酰基自由基 II 偶联得到远程硫砜基化产物 3a. 或者, PhS•先二聚生成 $\mathrm{PhSSPh}$, 它再与酰基自由基 II 反 应产生 3a. 在标准反应条件下, 的确可以观察到 $\mathrm{PhSSPh}$ 的生成。

\section{3 结论}

以硫代磺酸酯同时做砜基化和硫化试剂，实现了一 个可见光催化的烯烃砜基化启动的远程醛基碳-氢键直 接硫化反应，一步合成了 6-或 7-砜基取代的硫代羧酸 酯. 反应具有高度的原子经济性，室温进行，操作简单， 产率中等到良好, 底物普适性好, 预计具有良好的应用 前景. 初步的机理研究表明, 该反应经历了一个单电子 转移的自由基反应历程. 相比传统的烯烃 1,2-或 1,1-硫 砜基化反应，本文是首例官能团化烯烃的远程硫砜基化 反应，是对现有硫砜基化反应方法学的良好补充，为有 机硫化合物的合成提供了简单、高效和多样性的方法.

\section{4 实验部分}

操作步骤: 氮气氛下, 在 $25 \mathrm{~mL}$ 的 Schlenk 管中分 别加入 $2(0.5 \mathrm{mmol}), \operatorname{Ir}(\mathrm{ppy})_{3}(1 \mathrm{~mol} \%), \mathrm{K}_{2} \mathrm{HPO}_{4}(0.5$ $\mathrm{mmol}), \mathrm{MeCN}(4 \mathrm{~mL}), \mathbf{1}(0.25 \mathrm{mmol})$. 在 $15 \mathrm{~W}$ 的蓝色 LEDs 灯照射下 $25{ }^{\circ} \mathrm{C}$ 搅拌反应 $18 \mathrm{~h}$, 反应体系加水淬 灭, 接着用乙酸乙酯萃取, 无水硫酸钠干燥, 浓缩, 最 后硅胶柱层析分离得到产物 3 或 4.

\section{References}

[1] Madasu, S. B.; Vekariya, N. A.; Kiran, M. N. V. D. H.; Gupta, B.; Islam, A.; Douglas, P. S.; Babu, K. R. Beilstein J. Org. Chem. 2012 8,1400 .

[2] Fromtling, R. A. Drugs Future 1989, 14, 1165.

[3] Calverley, P. M. A.; Anderson, J. A.; Celli, B.; Ferguson, G. T.; Jenkins, C.; Jones, P. W.; Yates, J. C.; Vestbo, J. N. Engl. J. Med. 2007, 356,775 .

[4] (a) Chen, X.; Gan, X.; Chen, J.; Chen, Y.; Wang, Y.; Hu, D.; Song, B. Chin. J. Org. Chem. 2017, 37, 2343. (陈学文, 甘秀海, 陈吉祥, 
陈永中, 王艳娇, 胡德禹, 宋宝安, 有机化学, 2017, 37, 2343.) (b) Chen, Y.; Wang, S.; Jiang, Q.; Cheng, C.; Xiao, X.; Zhu, G. J. Org. Chem. 2018, 83, 716. For a review, see: (c) Feng, M.; Tang, B.; Liang, S. H.; Jiang, X. Curr. Top. Med. Chem. 2016, 16, 1200 and references cited therein.

[5] Julia, M.; Paris, J. M. Tetrahedron Lett. 1973, 14, 4833.

[6] Olah, G. A.; Mathew, T.; Prakash, G. K. S. Chem. Commun. 2001, 1696.

[7] (a) Deeming, A. S.; Russell, C. J.; Hennessy, A. J.; Willis, M. C. Org. Lett. 2014, 16, 150. (b) Wan, Y.; Zhang, J.; Chen, Y.; Kong, L.; Luo, F.; Zhu, G. Org. Biomol. Chem. 2017, 15, 7204.

[8] (a) Zhou, Q.; Gui, J.; Pan, C.-M.; Albone, E.; Cheng, X.; Suh, E. M.; Grasso, L.; Ishihara, Y.; Baran, P. S. J. Am. Chem. Soc. 2013, 135, 12994. (b) Miao, W.; Zhao, Y.; Ni, C.; Gao, B.; Zhang, W.; Hu, J. J. Am. Chem. Soc. 2018, 140, 880. (c) Griffiths, R. J.; Kong, W. C.; Richards, S. A.; Burley, G. A.; Willis, M. C.; Talbot, E. P. A. Chem. Sci. 2018, 9, 2295.

[9] For selected reviews, see: (a) Deeming, A. S.; Emmett, E. J.; Richards-Taylor, C. S.; Willis, M. C. Synthesis 2014, 46, 2701. (b) Liu, N.-W.; Liang, S.; Manolikakes, G. Synthesis 2016, 48, 1939. (c) Qiu, G.; Lai, L.; Cheng, J.; Wu, J. Chem. Commun. 2018, 54, 10405. (d) Guo, W.; Tao, K.; Tan, W.; Zhao, M.; Zheng, L.; Fan, X. Org. Chem. Front. 2019, 5, DOI: 10.1039/c8qo01353e. (e) Wang, S.; Zheng, Q.; Duan, P.; Liu, W. Chin. J. Org. Chem. 2017, 37, 1653. (王守锋, 郑 庆飞, 段盼盼, 刘文, 有机化学, 2017, 37, 1653.) (f) Tan, F.; Xiao, W.; Zeng, G. Chin. J. Org. Chem. 2017, 37, 824. (谭芬, 肖文精, 曾 国平, 有机化学, 2017, 37, 824.) (g) Li, S.; Hong, H.; Han, L.; Zhang, T.; Wang, Y.; Zhu, N. Chin. J. Org. Chem. 2018, 38, 304. (李 闪闪, 洪海龙, 韩利民, 张田苗, 王云龙, 矢宁, 有机化学, 2018, 38, 304.) (h) Li, S.-S.; Wang, J. Acta Chim. Sinica 2018, 76, 913 (in Chinese). (李树森, 王剑波, 化学学报, 2018, 76, 913.) For a report, see: (i) Yuan, Y.; Cao, Y.; Qiao, J.; Lin, Y.; Jiang, X.; Weng, Y.; Tang, S.; Lei, A. Chin. J. Chem. 2019, 37, 49.

[10] (a) Liu, T.; Li, Y.; Lai, L.; Cheng, J.; Sun, J.; Wu, J. Org. Lett. 2018, 20, 3605. (b) Ye, S.; Zheng, D.; Wu, J.; Qiu, G. Chem. Commun. 2019, 55, 2214.

[11] (a) Meyer, A. U.; Jäger, S.; Hari, D. P.; König, B. Adv. Synth. Catal. 2015, 357, 2050. (b) Zhang, G.; Zhang, L.; Yi, H. Luo, Y.; Qi, X.; Tung, C.-H.; Wu, L.-Z.; Lei, A. Chem. Commun. 2016, 52, 10407. (c) Ratushnyy, M.; Kamenova, M.; Gevorgyan, V. Chem. Sci. 2018, 9, 7193. (d) Sun, D.; Zhang, R. Org. Chem. Front. 2018, 5, 92. (e) Cai, S.; Xu, Y.; Chen, D.; Li, L.; Chen, Q.; Huang, M.; Weng, W. Org. Lett. 2016, 18, 2990.

[12] (a) Quebatte, L.; Thommes, K.; Severin, K. J. Am. Chem. Soc. 2006, 128, 7440. (b) Hossain, A.; Engl, S.; Lutsker, E.; Reiser, O. ACS Catal. 2019, 9, 1103. (c) Taniguchi, T.; Idota, A.; Ishibashi, H. Org. Biomol. Chem. 2011, 9, 3151. (d) Pagire, S. K.; Paria, S.; Reiser, O. Org. Lett. 2016, 18, 2106. (e) Xiong, Y.; Sun, Y.; Zhang, G. Org. Lett. 2018, 20, 6250. (f) Rao, W.-H.; Jiang, L.-L.; Liu, X.-M.; Chen, M.-J.; Chen, F.-Y.; Jiang, X.; Zhao, J.-X.; Zou, G.-D.; Zhou, Y.-Q.; Tang, L. Org. Lett. 2019, 21, 2890. (g) Wang, H.; Wang, G.; Lu, Q.; Chiang, C.-W.; Peng, P.; Zhou, J.; Lei, A. Chem. Eur. J. 2016, 22, 14489. (h) Yuan, Y.; Cao, Y.; Lin, Y.; Li, Y.; Huang, Z.; Lei, A. ACS Catal. 2018, 8, 10871.

[13] (a) Gao, Y.; Mei, H.; Han, J.; Pan, Y. Chem. Eur. J. 2018, 24, 17205. (b) Sun, J.; Li, P.; Guo, L.; Yu, F.; He, Y.-P.; Chu, L. Chem. Commun. 2018, 54, 3162. (c) Pirenne, V.; Kurtay, G.; Voci, S.; Bouffier, L.; Sojic, N.; Robert, F.; Bassani, D. M.; Landais, Y. Org. Lett. 2018, $20,4521$.

[14] (a) Chen, Z.-Z.; Liu, S.; Hao, W.-J.; Xu, G.; Wu, S.; Miao, J.-N.; Jiang, B.; Wang, S.-L.; Tu, S.-J.; Li, G. Chem. Sci. 2015, 6, 6654. (b)
Huang, M.-H.; Zhu, C.-F.; He, C.-L.; Zhu, Y.-L.; Hao, W.-J.; Wang, D.-C.; Tu, S.-J.; Jiang, B. Org. Chem. Front. 2018, 5, 1643. (c) Wu, W.; Yi, S.; Yu, Y.; Huang, W.; Jiang, H. J. Org. Chem. 2017, 82, 1224. (d) Cao, X.; Cheng, X.; Xuan, J. Org. Lett. 2018, $20,449$.

[15] (a) Zhu, D.; Shao, X.; Hong, X.; Lu, L.; Shen, Q. Angew. Chem., Int. Ed. 2016, 55, 15807. (b) Zhao, Q.; Lu, L.; Shen, Q. Angew. Chem., Int. Ed. 2017, 56, 11575.

[16] (a) Li, H.; Shan, C.; Tung, C.-H.; Xu, Z. Chem. Sci. 2017, 8, 2610. (b) Huang, S.; Thirupathi, N.; Tung, C.-H.; Xu, Z. J. Org. Chem. 2018, 83, 9449

[17] He, F.-S.; Wu, Y.; Zhang, J.; Xia, H.; Wu, J. Org. Chem. Front. 2018, $5,2940$.

[18] (a) Cheng, C.; Liu, S.; Lu, D.; Zhu, G. Org. Lett. 2016, 18, 2852. (b) Nie, X.; Cheng, C.; Zhu, G. Angew. Chem., Int. Ed. 2017, 56, 1898. (c) Jin, W.; Zhou, Y.; Zhao, Y.; Ma, Q.; Kong, L.; Zhu, G. Org. Lett. 2018, 20, 1435. (d) Wan, Y.; Shang, T.; Lu, Z. Zhu, G. Org. Lett. 2019, 21, 4187 .

[19] For selected reviews on photocatalysis, see: (a) Narayanam, J. M. R.; Stephenson, C. R. J. Chem. Soc. Rev. 2011, 40, 102. (b) Xuan, J.; Xiao, W.-J. Angew. Chem., Int. Ed. 2012, 51, 6828. (c) Prier, C. K.; Rankic, D. A.; MacMillan, D. W. C. Chem. Rev. 2013, 113, 5322. (d) Xi, Y.; Yi, H.; Lei, A. Org. Biomol. Chem. 2013, 11, 2387. (e) Yu, S.; Zhang, Y; Wang, R.; Jiang, H.; Cheng, Y; Kadi, A.; Fun, H.-K. Synthesis 2014, 2711. (f) Xie, J.; Jin, H.; Xu, P.; Zhu, C. Tetrahedron Lett. 2014, 55, 36. (g) Wang, C.; Lu, Z. Org. Chem. Front. 2015, 2 , 179. (h) Matsui, J. K.; Lang, S. B.; Heitz, D. R.; Molander, G. A. ACS Catal. 2017, 7, 2563.

[20] For selected reviews, see: (a) Hu, X.-Q.; Chen, J.-R.; Xiao, W.-J. Angew. Chem., Int. Ed. 2017, 56, 1960. (b) Li, W.; Xu, W.; Xie, J.; Yu, S.; Zhu, C. Chem. Soc. Rev. 2018, 47, 654. (c) Stateman, L. M.; Nakafuku, K. M.; Nagib, D. A. Synthesis 2018, 50, 1569. (d) Nechab, M.; Mondal, S.; Bertrand, M. P. Chem. Eur. J. 2014, 20, 16034 For selected reports involving 1,n-HAT since 2018, see: (e) Short, M. A.; Blackburn, J. M.; Roizen, J. L. Angew. Chem., Int. Ed. 2018 , 57, 296. (f) Dauncey, E. M.; Morcillo, S. P.; Douglas, J. J.; Sheikh, N. S.; Leonori, D. Angew. Chem., Int. Ed. 2018, 57, 744. (g) Wu, X.; Wang, M.; Huan, L.; Wang, D.; Wang, J.; Zhu, C. Angew. Chem., Int. Ed. 2018, 57, 1640. (h) Wu, S.; Wu, X.; Wang, D.; Zhu, C. Angew. Chem., Int. Ed. 2019, 58, 1499. (i) Jiang, H.; Studer, A. Angew. Chem., Int. Ed. 2018, 57, 1692. (j) Xia, Y.; Wang, L.; Studer, A. Angew. Chem., Int. Ed. 2018, 57, 12940. (k) Ratushnyy, M.; Parasram, M.; Wang, Y.; Gevorgyan, V. Angew. Chem., Int. Ed. 2018, 57, 2712. (1) Chuentragool, P.; Yadagiri, D.; Morita, T.; Sarkar, S.; Parasram, M.; Wang, Y.; Gevorgyan, V. Angew. Chem., Int. Ed. 2019, 58, 1794 (m) Na, C. G.; Alexanian, E. J. Angew. Chem., Int. Ed. 2018, 57, 13106. (n) Li, Z.; Wang, Q.; Zhu, J. Angew. Chem., Int. Ed. 2018, 57, 13288. (o) Bao, X.; Wang, Q.; Zhu, J. Angew. Chem., Int. Ed. 2019, 58, 2139. (p) Kim, I.; Park, B.; Kang, G.; Kim, J.; Jung, H.; Lee, H.; Baik, M.-H.; Hong, S. Angew. Chem., Int. Ed. 2018, 57, 15517. (q) Guan, H.; Sun, S.; Mao, Y.; Chen, L.; Lu, R.; Huang, J.; Liu, L. Angew. Chem., Int. Ed. 2018, 57, 11413. (r) Hu, A.; Guo, J.-J.; Pan, H.; Tang, H.; Gao, Z.; Zuo, Z. J. Am. Chem. Soc. 2018, 140, 1612. (s) An, X.-D.; Jiao, Y.-Y.; Zhang, H.; Gao, Y.; Yu, S. Org. Lett. 2018, 20, 401. (t) Zhu, Y.; Huang, K.; Pan, J.; Qiu, X.; Luo, X.; Qin, Q.; Wei, J.; Wen, X.; Zhang, L.; Jiao, N. Nat. Commun. 2018, 9 2625. (u) Li, G.-X.; Hu, X.; He, G.; Chen, G. Chem. Sci. 2019, 10, 688. (v) Zhang, Z.; Stateman, L. M.; Nagib, D. A. Chem. Sci. 2019, 10, 1207. (w) Wu, K.; Wang, L.; Colón-Rodríguez, S.; Flechsig, G.-U.; Wang, T. Angew. Chem., Int. Ed. 2019, 58, 1774. (x) Liu, Z.; Xiao, H.; Zhang, B.; Shen, H.; Zhu, L.; Li, C. Angew. Chem., Int. Ed. 2019, 58, 2510 . 